\title{
Long-term, interventional, open-label extension study evaluating the safety of tocilizumab treatment in patients with polyarticular-course juvenile idiopathic arthritis from Poland and Russia who completed the global, international CHERISH trial
}

\author{
Violetta Opoka-Winiarska ${ }^{1}$ Zbigniew Żuber ${ }^{2}$. Ekaterina Alexeeva ${ }^{3} \cdot{\text { Vyacheslav } \text { Chasnyk }^{4} \cdot \text { Irina Nikishina }}^{5}$. \\ Grażyna Dębowska $^{6}$ Elżbieta Smolewska ${ }^{7}$
}

Received: 14 January 2018 /Revised: 11 March 2018 / Accepted: 15 March 2018 / Published online: 13 April 2018

(C) The Author(s) 2018

\begin{abstract}
Efficacy and safety of tocilizumab (TCZ), an interleukin-6 receptor inhibitor, were demonstrated in juvenile idiopathic arthritis (JIA) with polyarticular course (pJIA) in the CHERISH trial. This observational, III phase study evaluated long-term treatment of TCZ in pJIA patients was conducted by members of the Pediatric Rheumatology International Trials Organization (PRINTO) from Poland and Russia. Forty-one patients, who had completed the CHERISH core study (104 weeks), were extensionally treated with TCZ ( $8 \mathrm{mg} / \mathrm{kg}$, intravenous infusion every 4 weeks). Total treatment time was from 131 to 193 weeks. The long-term safety (the primary endpoint) and efficacy were evaluated. All patients achieved ACR70 response in the core study and continued to achieve at least ACR50 response up to week 24 of this study. The safety population comprised 46.41 patient-years (PY). Rates per 100 PY of adverse (AEs) and serious events (SAEs) were 181.0 and 6.46, respectively. Pharyngitis and respiratory tract infections were the most common AEs. Except one AE (severe neutropenia), all others were classified as mild (24.4\%) or moderate $(29.3 \%)$. The incidence of SAEs was low (7.3\%). No new safety findings were observed. The safety profile of over 2.5-year treatment with TCZ is consistent with the pre-marketing CHERISH clinical trial. Presented data and continued efficacy response support the use of TCZ in pJIA. EUDRACT No: 2011-001607-12. https://clinicaltrials.gov/ct2/show/study/ NCT01575769?term=ML27783
\end{abstract}

Keywords Biologicals $\cdot$ Juvenile idiopathic arthritis $\cdot$ Tocilizumab $\cdot$ Treatment

Violetta Opoka-Winiarska

violetta.opoka-winiarska@umlub.pl

Zbigniew Żuber

zbyszekzuber@interia.pl

Ekaterina Alexeeva

alekatya@yandex.ru

Vyacheslav Chasnyk

chasnyk@gmail.com

Irina Nikishina

irpetnik@yandex.ru

Grażyna Dębowska

debowskag@gmail.com

Elżbieta Smolewska

e.smolewska@wp.pl
1 Department of Pediatric Pulmonology and Rheumatology, Medical University of Lublin, Gębali 6 Street, 20-093 Lublin, Poland

2 Department of Pediatric Neurology and Rheumatology, St. Louis Children's Hospital, Cracow, Poland

3 Federal State Autonomous Institution "National Medical Research Center of Children's Health" of the Ministry of Health of the Russian Federation, Federal State Autonomous Educational Institution of Higher Education I.M. Sechenov First Moscow State Medical University of the Ministry of Health of the Russian Federation, Moscow, Russia

4 St. Petersburg State Pediatric Medical Academy, St. Petersburg, Russia

5 Pediatric Department, Federal State Budgetary Institution V.A. Nasonova Research Institute of Rheumatology, Moscow, Russia

6 Medical Department, Roche Poland Sp. z o.o. (the employee of Roche Polska Sp. z o.o. till 30.04.2015), Warsaw, Poland

7 Department of Pediatric Rheumatology, Medical University of Lodz, Lodz, Poland 


\section{Introduction}

Juvenile idiopathic arthritis (JIA) is a clinically heterogeneous group of diseases characterized by arthritis that begins before 16 years of patient age and persists for minimum 6 weeks. The majority of children with JIA have a polyarticular form of the disease (pJIA) defined as arthritis in more than four joints during their disease course $[1,2]$. The course of pJIA is characterized by frequent relapses, long periods of active disease, higher risk of joint damage, low treatment effects, and reduced quality of life [2,3]. A variety of therapies are currently used in the treatment of pJIA, including both non-biologic and biologic disease modifying anti-rheumatic drugs (DMARDs) [4].

Tocilizumab (TCZ), a humanized, monoclonal anti-IL-6 receptor (IL-6R) antibody binding to membrane and soluble IL6R, inhibits IL-6-mediated signaling [4]. TCZ showed safety and activity in several studies in patients with systemic and polyarticular course of JIA [5-9]. Efficacy and safety of TCZ were demonstrated in the global, three-part, randomized, placebo-controlled, double-blind, withdrawal, phase III clinical trial (the CHERISH study) in patients with active pJIA (rheumatoid factor-positive or rheumatoid factor-negative $\mathrm{pJIA}$ or persisting oligoarticular JIA) for at least 6 months and inadequate response to methotrexate (MTX) [5]. The first part of the CHERISH study was a 16 -week open-label phase in which 188 patients received intravenous TCZ every 4 weeks. The improvement was evaluated by JIA ACR (the American College of Rheumatology) response. One hundred sixty-three patients, who achieved at least JIA ACR 30 responses, were enrolled into the second part of study. It was 24-week doubleblind phase with randomization 1:1 to placebo or to TCZ. The primary endpoint was JIA flare, compared with week 16. Patients flaring or completing part 2 received open-label TCZ up to week 104 of study. Published results of the CHERISH study demonstrated efficacy of TCZ therapy up to 40 weeks (parts 1 and 2) of treatment [5]. European Medicines Agency has approved TCZ to treat children with pJIA in 2013.

The purpose of the current analysis was to evaluate the longterm safety and efficacy of extensively prolonged TCZ therapy in 41 patients with pJIA from Poland and Russia who had completed the CHERISH core study. Total TCZ treatment time (with the core study) was from 131 to 193 weeks. Up to now, this is the longest published observation of patients with pJIA treated with TCZ.

\section{Methods}

\section{Patients and study design}

This long-term, interventional, open-label extension study evaluated the safety of TCZ treatment in patients with pJIA from Poland and Russia who completed the CHERISH study.
This study, as the core trial, was conducted by members of PRINTO from Poland and Russia. This was accordant with the Declaration of Helsinki, good clinical practice guidelines, local requirements, and approved by central IRB/IEC (Komisja Bioetyczna przy Uniwersytecie Medycznym w Lublinie, Poland).

The main inclusion criterion was the completion core, CHERISH study with at least JIA ACR 30 clinical response to TCZ with no adverse events (AEs), serious adverse events (SAEs), or conditions that could lead to unacceptable risk of continued treatment.

A total of 46 patients with pJlA from Poland and Russia who participated in the core CHERISH study were planned to be enrolled into this trial. Two patients from the CHERISH study refused further participation. Forty-three patients and parents agreed to continue the study and proceeded to screening. Two patients failed screening: one patient due to low neutrophil count and white blood cell count and one patient due to JIA ACR below 30 .

Written informed consent for the extension phase study participation was obtained from patients at the age 18 or older. For younger patients, written consent was signed by the one of the parents or legal guardian.

Finally, 41 patients were enrolled, 18 from Poland, and 23 from Russia. All those patients completed 104 weeks of treatment in the CHERISH study with at least JIA ACR 70 response to TCZ. Patients experienced no AEs, SAEs, or other conditions that could lead to the unacceptable risk of treatment continuation. Thus, all patients were eligible to continue the treatment (TCZ $8 \mathrm{mg} / \mathrm{kg}$ in intravenous infusion every 4 weeks) within this long-term extension study. Initially, the trial was planned for 104 weeks or until TCZ was commercially available for pJIA, whichever occurred first. The first TCZ dose in this regional extension protocol was to be administered at week 104 of the core study - at the last visit of the core study. All patients could have received ongoing MTX, non-steroidal anti-inflammatory drugs (NSAIDs), prednisone, or corticosteroid equivalents at the stable pre-entry doses during the study as the background medication. Patients treated with MTX also received either folic acid or folinic acid according to the local standard of care.

The extension study was terminated both in Russia and in Poland due to the fact that TCZ was approved in both countries for the new indication, i.e., pJIA. That was in accordance with the timeline provided in the study protocol and informed consent forms. As the result of the early termination, the follow-up period in extension study varied from 27 to 89 weeks. Total TCZ treatment time was therefore from 131 to 193 weeks (with the core study).

The long-term safety of TCZ treatment (the primary endpoint) was evaluated for the entire study process (27 to 89 weeks depend on the patient) and follow-up visit. For the purpose of efficacy endpoint analysis, data for the first 
24 weeks in which all patients remained in the study are presented in this report. Efficacy was assessed in relation to the first visit in the core study. There, adding together, our study assesses the effectiveness of the entire TCZ treatment process lasting a total of 128 weeks. Later time points are referenced only. The publication the CHERISH study presented efficacy results of the first 40 weeks and safety results of entire study [5].

\section{Assessment and outcomes}

Clinical assessments (baseline and every 4 weeks thereafter) included six JIA core response variables: number of joints with active arthritis, number of joints with limitation of motion (LOM), physician global assessment (PGA) of disease activity (range, 0-100), assessment of patient overall well-being (range, 0-100), physical function measured by the Childhood Health Assessment Questionnaire-Disability Index (CHAQ-DI; range, 0-3), and erythrocyte sedimentation rate (ESR) [10]. Clinically inactive disease was defined as PGA indicating no disease activity and the absence of all of the following: joints with active arthritis, uveitis, and ESR greater than $20 \mathrm{~mm} / \mathrm{h}$ [11].

\section{The primary endpoints}

The primary endpoint was to evaluate the long-term safety of TCZ treatment in patients with pJIA who entered this extension study. The primary safety outcome measures included the number and percentage of AEs, SAEs, AEs of special interest, and study drug-related AEs. Other safety endpoints included clinical laboratory evaluations, physical examination, vital signs, height/weight, electrocardiography (ECG), tuberculosis (TB) test, chest X-ray, slit-lamp ophthalmology examination, and recording of hospitalizations/deaths. A tuberculin test, either protein derivative tuberculin skin test (PPD) or interferonbased test and chest X-ray, was performed yearly during the whole period of TCZ treatment, due to safety reasons. Pneumococcal vaccination has not been performed in the study group.

\section{Secondary endpoints}

The secondary endpoint was to investigate the long-term efficacy during treatment with TCZ in the study group.

The efficacy was assessed in relation to the first visit of the core study. The following parameters were evaluated at visits: baseline (during the last visit of core study) and every 4 weeks thereafter to 24 week of study:

- The proportion of patients with JIA ACR responses. The JIA ACR30/50/70/90 response was defined as three of any six core outcome variables improved by at least $30 \% / 50 \% / 70 \% / 90 \%$ from the baseline assessments, with no more than one of the remaining variables worsened by more than $30 \%$. Patients were required to have at least JIA ACR 30 clinical response to TCZ at week 104 of the core study to continue to this extension study.

- The proportion of patients with inactive disease and clinical remission (CR) at visits.

Four levels of CR were defined for patients during TCZ treatment in this study:

Level 1: $\quad$ CR on medication for a minimum of 6 consecutive months, while the patient was on medication

Level 2: CR off corticosteroid medication (still on TCZ) for a minimum of 6 consecutive months, while the patient was off corticosteroid medications

Level 3: CR off both corticosteroid and MTX medication (still on TCZ) for a minimum of 6 consecutive months, while the patient was off both MTX and corticosteroids

Level 4: CR off all anti-inflammatory medications (still on TCZ) for a minimum of 6 consecutive months, while the patient was off all anti-arthritis medications (corticosteroids, MTX, NSAIDs).

\section{Statistics}

All statistical analyses were conducted using Statistical Analysis System (SAS ${ }^{\circ}$ ) release 9.1.3. As no formal hypothesis was tested, intent-to-treat and per-protocol populations were not defined for this study. Laboratory data were summarized using mean, standard deviation (SD), median, minimum, and maximum of the actual values at each assessment. The primary and secondary analyses were conducted using the safety population defined as all patients who entered the study, took at least one dose of the study drug, and had at least one safety follow-up regardless of premature withdrawal.

Patient data for visit 1 week 0 (the baseline) of current study was taken from 33 visit week 104 of core study.

For some data summaries, the baseline values from the core study were used. Analysis in tables and listings are presented overall and by country. AE data were summarized using counts and percentages overall, by intensity, and by relationship to the study drug. Summaries of deaths, withdrawals from study drug, and marked laboratory abnormalities were also presented using counts and percentages.

\section{Results}

\section{Demographics and baseline disease characteristics}

The demographics and baseline disease characteristics were comparable with the pediatric patient population from 
the core study (CHERISH study). The mean age was 12.0 \pm 4.45 years with a range from 4 to 19 years. All patients were white and $80.5 \%$ were females (Table 1). Differences in age were reflected in the range of height, weight, and body mass index (BMI). All patients weighed at least $30 \mathrm{~kg}$. The mean duration of pJIA was $4.8 \pm 4.08$ years. All patients in this study achieved ACR70 at baseline (week 104 of the core study). Demographics and baseline disease characteristics were generally similar in patients from Poland as well as from Russia.

Concomitant medications were reported for all 18 patients $(100 \%)$ in Poland and in 16 patients $(69.6 \%)$ in Russia (Table 2). MTX was the most frequently reported medication (29 patients, $70.7 \%$ ). In this study, as in the core CHERISH study, the stable doses of MTX $(<20-\mathrm{mg} /$ $\mathrm{m}^{2}$ body surface area/week), NSAIDs and low-dose glucocorticoids (no greater than $0.2-\mathrm{mg} / \mathrm{kg} /$ day prednisone; daily maximum, $10 \mathrm{mg}$ ) were allowed. No other disease modifying drugs were used during the study. The MTX dose reduction depended on the attending physician. The dose might be decreased due to safety reasons or for efficacy in patients who have been in CR for a minimum of 6 months and remained off all corticosteroids.

\section{Safety evaluation}

\section{Extent of the exposure}

The overall mean duration of exposure to TCZ in the study was $55.8 \pm 19.40$ weeks, together with core study, $159.8 \pm$ 19.40 weeks), and the mean number of infusions was $14.3 \pm$ 4.76 (with core study $47.3 \pm 4.76$ ). The total patient duration in the study was 46.41 years (158.41 years with core study).

\section{Adverse events}

Event rates per 100 patient-years (PY) of exposure were retrospectively calculated for AEs, serious AEs, and infection AEs. Overall, 23 patients (56.1\%) experienced 84 events during the study (the event rate of 181.00 AEs per $100 \mathrm{PY}$ ). They are presented in Table 3.

Adverse events by severity The majority of patients had AEs that were mild (10 patients, $24.4 \%$ ) or moderate (12 patients, $29.3 \%)$ in intensity, and one patient $(2.4 \%)$ experienced a severe AE of the decreased neutrophil count (Table 3). Pharyngitis (14.6\%) and upper respiratory tract infections
Table 1 Demographics and other baseline disease characteristics (safety population)

\begin{tabular}{llll}
\hline Characteristics & Poland $(N=18)$ & Russia $(N=23)$ & All Patients $(N=41)$ \\
\hline Age (years), mean (SD) & $12.9(4.70)$ & $11.3(4.20)$ & $12.0(4.45)$ \\
Females, $n(\%)$ & $16(88.9)$ & $17(73.9)$ & $33(80.5)$ \\
White race, $n(\%)$ & $18(100)$ & $23(100)$ & $41(100)$ \\
Height (cm), mean (SD) & $149.7(20.25)$ & $149.6(22.5)$ & $149.6(21.29)$ \\
$\quad$ Median & 158.8 & 159.0 & 159.0 \\
Min, max & $109.0,174.0$ & $110.0,184.5$ & $109.0,184.5$ \\
Weight (kg), mean (SD) & $44.6(16.03)$ & $43.3(19.24)$ & $43.9(17.70)$ \\
$\quad$ Median & 49.6 & 44.0 & 46.3 \\
Min, max & $17.6,65.0$ & $15.5,89.7$ & $15.5,89.7$ \\
Body mass index, mean (SD) & $19.2(3.70)$ & $18.3(3.91)$ & $18.7(3.80)$ \\
Median & 19.4 & 17.4 & 18.1 \\
Min, max & $14.1,27.6$ & $12.8,31.4$ & $12.8,31.4$ \\
Disease duration (months), mean (SD) & $50.9(35.53)$ & $62.6(57.65)$ & $57.5(48.97)$ \\
Joints with active arthritis, mean (SD) & $1.3(2.02)$ & $1.6(3.15)$ & $1.4(2.68)$ \\
Joints with LOM, mean (SD) & $2.8(5.86)$ & $4.0(6.71)$ & $3.5(6.30)$ \\
PtGA VAS, mean (SD) & $4.7(7.65)$ & $3.4(3.65)$ & $4.0(5.72)$ \\
PGA, mean (SD) & $5.6(6.24)$ & $3.7(3.67)$ & $4.5(4.99)$ \\
CHAQ-DI, mean (SD) & $0.14(0.283)$ & $0.14(0.209)$ & $0.14(0.241)$ \\
ESR, mean (SD) & $6.20(5.370)$ & $3.90(2.249)$ & $4.95(4.084)$ \\
CRP, mean (SD) & $1.83(6.107)$ & $0.22(0.118)$ & $0.95(4.114)$ \\
\hline
\end{tabular}

Abbreviations: ACR = American College of Rheumatology; CHAQ-DI = Childhood Health Assessment Questionnaire Disability Index; CRP = C-reactive protein; ESR = erythrocyte sedimentation rate; JIA = juvenile idiopathic arthritis; LOM = limitation of movement; PGA = physician global assessment; PtGA = patient/parent global assessment; $\mathrm{SD}=$ standard deviation; $\mathrm{VAS}=$ visual analog scale; body mass index $=$ weight $(\mathrm{kg}) /($ height $(\mathrm{m}))^{2}$

Demographic data were taken from visit 33 (week 104) of the core study 
Table 2 Concomitant background medication (MTX, NSAIDs or corticosteroids)

\begin{tabular}{llll}
\hline & $\begin{array}{l}\text { Poland }(N=18) \\
n(\%)\end{array}$ & $\begin{array}{l}\text { Russia }(N=23) \\
n(\%)\end{array}$ & $\begin{array}{l}\text { All patients }(N=41) \\
n(\%)\end{array}$ \\
\hline $\begin{array}{l}\text { Number of patients reporting any concomitant } \\
\text { background medication (MTX, NSAIDs } \\
\text { or corticosteroids) }\end{array}$ & $18(100)$ & $15(65.2)$ & $33(80.5)$ \\
$\begin{array}{l}\text { Concomitant MTX } \\
\text { MTX dose summary (mg/week) }\end{array}$ & $16(88.9)$ & $13(56.5)$ & $29(70.7)$ \\
Mean & & & \\
95\% CI & 11.25 & 10.97 & 11.15 \\
SD & $9.42,13.08$ & $9.26,12.68$ & $9.87,12.42$ \\
Minimum & 4.541 & 3.085 & 4.030 \\
Median & 2.5 & 6.0 & 2.5 \\
Maximum & 10.00 & 10.00 & 10.00 \\
Concomitant NSAIDs & 20.0 & 15.0 & 20.0 \\
Concomitant corticosteroids & $17(94.4)$ & $9(39.1)$ & $26(63.4)$ \\
Corticosteroid dose summary & $5(27.8)$ & 0 & $5(12.2)$ \\
Mean & & & \\
95\% CI & 4.25 & $\mathrm{NC}$ & 4.25 \\
SD & 5.47 & $\mathrm{NC}$ & 3.03 \\
Minimum & 1.591 & $\mathrm{NC}$ & 1.591 \\
Median & 2.5 & $\mathrm{NC}$ & 2.5 \\
Maximum & 4.00 & $\mathrm{NC}$ & 4.00 \\
\hline
\end{tabular}

Abbreviations: $\mathrm{ATC}=$ anatomical therapeutic chemical classification; $\mathrm{CI}=$ confidence interval; $\mathrm{MTX}=$ methotrexate; $\mathrm{NC}=$ not calculable, NSAIDs = non-steroidal anti-inflammatory drugs; $\mathrm{SD}=$ standard deviation; $\mathrm{WHO}=$ World Health Organization

WHO drug dictionary version March 2012

Some corticosteroids doses were adjusted to be prednisone equivalent doses
(12.2\%) were the most frequently AEs occurring. There were three SAEs $(7.3 \%, 6.46$ per $100 \mathrm{PY})$ : mild proteinuria, severe neutrophil count decreased, and multiple injuries of moderate severity. There were no deaths, life-threatening AEs (especially malignancies, active TB, or demyelinating disorders) or AEs of special interest during this study. There were a small number of patients with clinically significant abnormal laboratory parameters, the majority of which were isolated cases. One patient had significantly increased liver function tests at the follow-up visit following a reported AE of Epstein-Barr virus infection. Mean values of bilirubin, total cholesterol, low-density lipoprotein cholesterol, high-density lipoprotein cholesterol, and triglyceride concentrations remained stable throughout the study.

Adverse events by relationship to the study drug Overall, there were 12 patients $(29.3 \%)$ who experienced drugrelated AEs that were remotely, probably, or possibly related to TCZ. There were two patients with AEs considered possibly related to TCZ: mild proteinuria and mild decrease in hemoglobin concentration. Both AEs resolved within 5 weeks did not lead to any dose change of the study drug and patients continued the study. The SAE of severe neutrophil count decreased was considered probably related to TCZ; the remaining two SAEs (mild proteinuria and multiple injuries) were considered unrelated.

Nine patients (22.0\% of all patients) had an AE that led to dose modifications. There was one patient withdrawn from the treatment due to an AE during the study (severe decrease in neutrophil count). In the remaining eight patients, dose of the drug was omitted due to respiratory tract infections (five patients), dermatitis (two patients), and hyperbilirubinemia (one patient).

\section{Efficacy evaluation}

\section{Proportion of patients with JIA ACR 30/50/70/90 responses}

Proportions of patients sustaining ACR response at time points to visit 7 (week 24) are presented in the Table 4. All patients in this study achieved ACR70 at baseline (week 104 of the core study). Overall, all patients maintained ACR50 response (using baseline visit of the core study) between baseline (week 104 of the core study) and visit 7 (week 24). Forty patients (97.6\%) from 41 treated with TCZ maintained ACR70 response during the study. Only one patient presented the decrease in the level of response from ACR 70 to ACR 50. 
Table 3 Serious adverse events and adverse events occurring in at least 5\% of the patients by treatment group for events

\begin{tabular}{|c|c|c|c|c|c|}
\hline Preferred term & $\begin{array}{l}\text { Mild } \\
n(\%) \mathrm{E}\end{array}$ & $\begin{array}{l}\text { Moderate } \\
n(\%) \mathrm{E}\end{array}$ & $\begin{array}{l}\text { Severe } \\
n(\%) \mathrm{E}\end{array}$ & $\begin{array}{l}\text { Life-threatening } \\
n(\%) \mathrm{E}\end{array}$ & $\begin{array}{l}\text { Total } \\
n(\%) \mathrm{E}\end{array}$ \\
\hline Patients with any AEs & $10(24.4) 61$ & $12(29.3) 22$ & $1(2.4) 1$ & 0 & $23(56.1) 84$ \\
\hline Peripheral blood & $2(4.9) 5$ & $1(2.4) 2$ & 0 & 0 & $3(7.3) 7$ \\
\hline Neutropenia & $2(4.9) 3$ & $1(2.4) 1$ & 0 & 0 & $3(7.3) 4$ \\
\hline Leukopenia & $1(2.4) 2$ & $1(2.4) 1$ & 0 & 0 & $2(4.9) 3$ \\
\hline Eye disorders & $2(4.9) 2$ & 0 & 0 & 0 & $2(4.9) 2$ \\
\hline Corneal erosion & $1(2.4) 1$ & 0 & 0 & & $1(2.4) 1$ \\
\hline Corneal opacity & $1(2.4) 1$ & 0 & 0 & 0 & $1(2.4) 1$ \\
\hline Gastrointestinal disorders & $2(4.9) 2$ & $1(2.4) 1$ & 0 & 0 & $3(7.3) 3$ \\
\hline Dyspepsia & $1(2.4) 1$ & 0 & 0 & 0 & $1(2.4) 1$ \\
\hline Periodontitis & 0 & $1(2.4) 1$ & 0 & 0 & $1(2.4) 1$ \\
\hline Vomiting & $1(2.4) 1$ & 0 & 0 & 0 & $1(2.4) 1$ \\
\hline Infections and infestations & $8(19.5) 26$ & $11(26.8) 15$ & 0 & 0 & 19 (46.3) 41 \\
\hline Pharyngitis & $4(9.8) 6$ & $2(4.9) 2$ & 0 & 0 & $6(14.6) 8$ \\
\hline Upper RTI & $3(7.3) 6$ & $2(4.9) 3$ & 0 & 0 & $5(12.2) 9$ \\
\hline Bronchitis & $1(2.4) 1$ & $3(7.3) 3$ & 0 & 0 & $4(9.8) 4$ \\
\hline Acute sinusitis & 0 & $2(4.9) 3$ & 0 & 0 & $2(4.9) 3$ \\
\hline Rhinitis & $2(4.9) 2$ & 0 & 0 & 0 & $2(4.9) 2$ \\
\hline Viral upper RTI & $1(2.4) 1$ & $1(2.4) 1$ & 0 & 0 & $2(4.9) 2$ \\
\hline Blister infected & 0 & $1(2.4) 1$ & 0 & 0 & $1(2.4) 1$ \\
\hline Mononucleosis & 0 & $1(2.4) 1$ & 0 & 0 & $1(2.4) 1$ \\
\hline Oral herpes & $1(2.4) 1$ & 0 & 0 & 0 & $1(2.4) 1$ \\
\hline Otitis media & $1(2.4) 1$ & 0 & 0 & 0 & $1(2.4) 1$ \\
\hline Paronychia & $1(2.4) 1$ & 0 & 0 & 0 & $1(2.4) 1$ \\
\hline Pharyngotonsillitis & 0 & $1(2.4) 1$ & 0 & 0 & $1(2.4) 1$ \\
\hline RTI & $1(2.4) 1$ & 0 & 0 & 0 & $1(2.4) 1$ \\
\hline Tinea versicolour & $1(2.4) 1$ & 0 & 0 & 0 & $1(2.4) 1$ \\
\hline Tonsillitis & $1(2.4) 1$ & 0 & 0 & 0 & $1(2.4) 1$ \\
\hline Urinary infection & $1(2.4) 1$ & 0 & 0 & 0 & $1(2.4) 1$ \\
\hline Varicella & $1(2.4) 1$ & 0 & 0 & 0 & $1(2.4) 1$ \\
\hline Viral infection & $1(2.4) 1$ & 0 & 0 & 0 & $1(2.4) 1$ \\
\hline Viral rhinitis & $1(2.4) 1$ & 0 & 0 & 0 & $1(2.4) 1$ \\
\hline Investigations & $2(4.9) 9$ & 0 & $1(2.4) 1$ & 0 & $3(7.3) 10$ \\
\hline$\uparrow \mathrm{ALT}$ & $1(2.4) 1$ & 0 & 0 & 0 & $1(2.4) 1$ \\
\hline$\uparrow$ Bilirubin c. & $1(2.4) 2$ & 0 & 0 & 0 & $1(2.4) 2$ \\
\hline$\uparrow$ Bilirubin & $1(2.4) 2$ & 0 & 0 & 0 & $1(2.4) 2$ \\
\hline$\uparrow \mathrm{CRP}$ & $1(2.4) 1$ & 0 & 0 & 0 & $1(2.4) 1$ \\
\hline$\downarrow \mathrm{Hb}$ & $1(2.4) 1$ & 0 & 0 & 0 & $1(2.4) 1$ \\
\hline$\downarrow \mathrm{Neu}$ & 0 & 0 & $1(2.4) 1$ & 0 & $1(2.4) 1$ \\
\hline$\uparrow \mathrm{ESR}$ & $1(2.4) 1$ & 0 & 0 & 0 & $1(2.4) 1$ \\
\hline$\downarrow \mathrm{WBC}$ & $1(2.4) 1$ & 0 & 0 & 0 & $1(2.4) 1$ \\
\hline Metabolism/nutrition disorders & $2(4.9) 2$ & 0 & 0 & 0 & $2(4.9) 2$ \\
\hline Hypercholesterolemia & $1(2.4) 1$ & 0 & 0 & 0 & $1(2.4) 1$ \\
\hline Hypertriglyceridemia & $1(2.4) 1$ & 0 & 0 & 0 & $1(2.4) 1$ \\
\hline Musculoskeletal/connective tissue disorders & $2(4.9) 2$ & $1(2.4) 1$ & 0 & 0 & $3(7.3) 3$ \\
\hline Back pain & 0 & $1(2.4) 1$ & 0 & 0 & $1(2.4) 1$ \\
\hline Joint swelling & $1(2.4) 1$ & 0 & 0 & 0 & $1(2.4) 1$ \\
\hline Osteoarthritis & $1(2.4) 1$ & 0 & 0 & 0 & $1(2.4) 1$ \\
\hline Nervous system disorders & $1(2.4) 1$ & $1(2.4) 1$ & 0 & 0 & $2(4.9) 2$ \\
\hline
\end{tabular}


Table 3 (continued)

\begin{tabular}{|c|c|c|c|c|c|}
\hline Preferred term & $\begin{array}{l}\text { Mild } \\
n(\%) \mathrm{E}\end{array}$ & $\begin{array}{l}\text { Moderate } \\
n(\%) \mathrm{E}\end{array}$ & $\begin{array}{l}\text { Severe } \\
n(\%) \mathrm{E}\end{array}$ & $\begin{array}{l}\text { Life-threatening } \\
n(\%) \mathrm{E}\end{array}$ & $\begin{array}{l}\text { Total } \\
n(\%) \mathrm{E}\end{array}$ \\
\hline Headache & $1(2.4) 1$ & $1(2.4) 1$ & 0 & 0 & $2(4.9) 2$ \\
\hline Renal/urinary disorders & $2(4.9) 6$ & 0 & 0 & 0 & $2(4.9) 6$ \\
\hline Proteinuria & $2(4.9) 3$ & 0 & 0 & 0 & $2(4.9) 3$ \\
\hline Hematuria & $1(2.4) 1$ & 0 & 0 & 0 & $1(2.4) 1$ \\
\hline Leukocyturia & $1(2.4) 2$ & 0 & 0 & 0 & $1(2.4) 2$ \\
\hline
\end{tabular}

Abbreviations: $\mathrm{AE}=$ adverse event $\mathrm{E}=$ number of events; MedDRA = medical dictionary for regulatory activities, $\mathrm{PT}=\mathrm{preferred}$ term, $\mathrm{SOC}=\mathrm{system}$ organ class, $\mathrm{RTI}=$ respiratory tract infection, $\mathrm{ALT}=$ alanine aminotransferase, Bilirubin $\mathrm{c} .=$ bilirubin conjugated, $\mathrm{CRP}=\mathrm{C}-$ reactive protein, $\mathrm{Hb}=$ hemoglobin, $\mathrm{Neu}=$ neutrophil count, $\mathrm{ESR}=$ erythrocyte sedimentation rate, $\mathrm{WBC}=$ white blood cell count

MedDRA V15.0 coding dictionary

If a patient experienced the same $\mathrm{AE}$ preferred term but with different severities, they were counted in the most extreme category only. However, the multiple events of the same AE preferred term were counted in each severity that they occurred. Hence, the total number of events for any one preferred term may exceed the sum of events within individual categories where patient counts exist

\section{Proportion of patients with inactive disease at visits}

The proportion of patients with inactive disease at selected visits is provided in Table 5. Overall, the number of patients who achieved inactive disease increased between baseline and visit 7 (week 24) from 26 patients (63.4\%) to 31 (75.6\%), respectively.

\section{Proportion of patients with clinical remission at visits}

The proportion of patients with $\mathrm{CR}$ at time points to visit 7 (week 24 ) is provided in Table 6. Overall, the number of patients who achieved CR increased between baseline and visit 7 (week 24) from $18(43.9 \%)$ patients to $20(48.8 \%)$, respectively. The most frequent level of the response observed was level 1 and level 2.

\section{Discussion}

Results of this first observational, long-term study on TCZ in patients with pJIA demonstrate that continuing treatment over 104 to 131 weeks or longer with intravenous TCZ $(8 \mathrm{mg} / \mathrm{kg}$ administered every 4 weeks) is safe and efficacious for the

Table 4 Proportion of patients with JIA ACR30/50/70/90 responses by visit (response calculated based on baseline visit of the core study)

\begin{tabular}{|c|c|c|c|c|}
\hline Visit & JIA ACR response & $\begin{array}{l}\text { Poland }(N=18) \\
n(\%) 95 \% \mathrm{CI}^{\mathrm{a}}\end{array}$ & $\begin{array}{l}\text { Russia }(N=23) \\
n(\%) 95 \% \mathrm{CI}^{\mathrm{a}}\end{array}$ & $\begin{array}{l}\text { All patients }(N=41) \\
n(\%) 95 \% \mathrm{Cl}^{\mathrm{a}}\end{array}$ \\
\hline \multicolumn{5}{|c|}{ Visit 1: week 0 (baseline $^{\mathrm{b}}$ ) } \\
\hline & ACR 30 response & 18 (100) 81.5100 .0 & 23 (100) 85.2100.0 & 41 (100) 91.4, 100.0 \\
\hline & ACR50 response & 18 (100) 81.5, 100.0 & 23 (100) 85.2, 100.0 & 41 (100) 91.4, 100.0 \\
\hline & ACR70 response & 18 (100) 81.5, 100.0 & 23 (100) 85.2, 100.0 & 41 (100) 91.4, 100.0 \\
\hline & ACR90 response & $11(61.1) 35.7,82.7$ & 19 (82.6) 61.2, 95.0 & 30 (73.2) 57.1, 85.8 \\
\hline \multicolumn{5}{|c|}{ Visit 4: week 12} \\
\hline & ACR 30 response & 18 (100) 81.5, 100.0 & 23 (100) 85.2, 100.0 & 41 (100) 91.4, 100.0 \\
\hline & ACR50 response & 18 (100) 81.5, 100.0 & 23 (100) 85.2, 100.0 & 41 (100) 91.4, 100.0 \\
\hline & ACR70 response & 18 (100) 81.5, 100.0 & 23 (100) 85.2, 100.0 & 41 (100) 91.4, 100.0 \\
\hline & ACR90 response & $12(66.7) 41.0,86.7$ & $20(87.0) 66.4,97.2$ & $32(78.0) 62.4,89.4$ \\
\hline \multicolumn{5}{|c|}{ Visit 7: week 24} \\
\hline & ACR 30 response & 18 (100) 81.5, 100.0 & 23 (100) 85.2, 100.0 & 41 (100) 91.4, 100.0 \\
\hline & ACR50 response & 18 (100) 81.5, 100.0 & 23 (100) 85.2, 100.0 & 41 (100) 91.4, 100.0 \\
\hline & ACR70 response & 17 (94.4) 72.7, 99.9 & 23 (100) 85.2, 100.0 & 40 (97.6) 87.1, 99.9 \\
\hline & ACR90 response & 13 (72.2) 46.5, 90.3 & $20(87.0) 66.4,97.2$ & 33 (80.5) 65.1, 91.2 \\
\hline
\end{tabular}

Abbreviations: $\mathrm{ACR}=$ American College of Rheumatology; $\mathrm{CI}=$ confidence interval; JIA = juvenile idiopathic arthritis

${ }^{\mathrm{a}}$ Exact 2-sided CI (Clopper and Pearson) based upon the observed proportion of patients

${ }^{\mathrm{b}}$ Baseline is the baseline visit of study and uses visit 33 (week 104) of the core study. For some patients, re-screening value was used as baseline to consider the last available non-missing value prior to the start of treatment 
Table 5 Proportion of patients with inactive disease by visit (safety population)

\begin{tabular}{llll}
\hline Visit & $\begin{array}{l}\text { Poland }(N=18) \\
n(\%) 95 \% \mathrm{CI}^{\mathrm{a}}\end{array}$ & $\begin{array}{l}\text { Russia }(N=23) \\
n(\%) 95 \% \mathrm{CI}^{\mathrm{a}}\end{array}$ & $\begin{array}{l}\text { All patients }(N=41) \\
n(\%) 95 \%\end{array}$ \\
\hline $\begin{array}{l}\text { Visit 1: week 0 (baseline) } \\
\quad \text { Inactive disease }\end{array}$ & $11(61.1) 35.7,82.7$ & $15(65.2) 42.7,83.6$ & $26(63.4) 46.9,77.9$ \\
$\quad$ Signs of still active disease & $7(38.9) 17.3,64.3$ & $8(34.8) 16.4,57.3$ & $15(36.6) 22.1,53.1$ \\
Visit 4: week 12 & $11(61.1) 35.7,82.7$ & $16(69.6) 47.1,86.8$ & $27(65.9) 49.4,79.9$ \\
$\quad$ Inactive disease & $7(38.9) 17.3,64.3$ & $7(30.4) 13.2,52.9$ & $14(34.1) 20.1,50.6$ \\
$\quad \begin{array}{l}\text { Signs of still active disease } \\
\text { Visit 7: week 24 }\end{array}$ & $11(61.1) 35.7,82.7$ & $20(87.0) 66.4,97.2$ & $31(75.6) 59.7,87.6$ \\
$\quad \begin{array}{l}\text { Inactive disease } \\
\text { Signs of still active disease }\end{array}$ & $7(38.9) 17.3,64.3$ & $3(13.0) 2.8,33.6$ & $10(24.4) 12.4,40.3$ \\
\hline
\end{tabular}

Abbreviations: $\mathrm{CI}=$ confidence interval;

Week 0 data were taken from visit 33 (week 104) of the core study

${ }^{\text {a }}$ Exact 2-sided CI (Clopper and Pearson) based upon the observed proportion of patients management of pJIA. The safety and efficacy of TCZ for the treatment of pJIA were demonstrated in several only in few studies, although their follow-up periods were shorter than in our study [5-7].

The overall incidence of $\mathrm{AE}$ and SAEs rates during the follow-up period of this study was found to be lower to the results of the core study, 181.0 and 6.46 , respectively. The event rate of infection AEs was 88.34/100 PY. Pharyngitis and upper respiratory tract infection were the most common
AE: 14.6 and $12.2 \%$, respectively, in the core study 10.1 and $9.0 \%$, respectively. The frequency of neutropenia was $7.3 \%$, in core study $3.7 \%$. Hypercholesterolemia occurred in one child, whereas in the core study in $34.6 \%$. With the exception of one patient who had severe neutropenia that led to withdrawal from the study, all other AEs were either mild (24.4\%) or moderate $(29.3 \%)$. There were no deaths, life-threatening AEs, or AEs of specific side effect. The majority of AEs were unrelated to the study drug.
Table 6 Proportion of patients with clinical remission by visit (safety population)

\begin{tabular}{|c|c|c|c|}
\hline Visit & $\begin{array}{l}\text { Poland }(N=18) \\
n(\%) 95 \% \mathrm{CI}^{\mathrm{a}}\end{array}$ & $\begin{array}{l}\text { Russia }(N=23) \\
n(\%) 95 \% \mathrm{CI}^{\mathrm{a}}\end{array}$ & $\begin{array}{l}\text { All patients }(N=41) \\
n(\%) 95 \% \mathrm{CI}^{\mathrm{a}}\end{array}$ \\
\hline \multicolumn{4}{|c|}{ Visit 1: week 0 (baseline) } \\
\hline Clinical remission & 8 (44.4) $21.5,69.2$ & $10(43.5) 23.2,65.5$ & 18 (43.9) 28.5, 60.3 \\
\hline Level 1 & $1(5.6) 0.1,27.3$ & $2(8.7) 1.1,28.0$ & $3(7.3) 1.5,19.9$ \\
\hline Level 2 & $7(38.9) 17.3,64.3$ & 6 (26.1) $10.2,48.4$ & 13 (31.7) 18.1, 48.1 \\
\hline Level 3 & $00.0,18.5$ & $1(4.3) 0.1,21.9$ & $1(2.4) 0.1,12.9$ \\
\hline Level 4 & $00.0,18.5$ & $1(4.3) 0.1,21.9$ & $1(2.4) 0.1,12.9$ \\
\hline \multicolumn{4}{|l|}{ Visit 4: week 12} \\
\hline Clinical remission & $9(50.0) 26.0,74.01$ & 0 (43.5) 23.2, 65.5 & 19 (46.3) 30.7, 62.6 \\
\hline Level 1 & $2(11.1) 1.4,34.7$ & $2(8.7) 1.1,28.0$ & $4(9.8) 2.7,23.1$ \\
\hline Level 2 & 7 (38.9) 17.3, 64.3 & 6 (26.1) $10.2,48.4$ & 13 (31.7) 18.1, 48.1 \\
\hline Level 3 & $00.0,18.5$ & $1(4.3) 0.1,21.9$ & $1(2.4) 0.1,12.9$ \\
\hline Level 4 & $00.0,18.5$ & $1(4.3) 0.1,21.9$ & 1 (2.4) $0.1,12.9$ \\
\hline \multicolumn{4}{|l|}{ Visit 7: week 24} \\
\hline Clinical remission & 10 (55.6) $30.8,78.5$ & 10 (43.5) 23.2, 65.5 & 20 (48.8) 32.9, 64.9 \\
\hline Level 1 & 3 (16.7) 3.6, 41.4 & $2(8.7) 1.1,28.0$ & 5 (12.2) 4.1, 26.2 \\
\hline Level 2 & $7(38.9) 17.3,64.3$ & 6 (26.1) 10.2, 48.4 & 13 (31.7) 18.1, 48.1 \\
\hline Level 3 & $00.0,18.5$ & $1(4.3) 0.1,21.9$ & $1(2.4) 0.1,12.9$ \\
\hline Level & $00.0,18.5$ & $1(4.3) 0.1,21.9$ & $1(2.4) 0.1,12.9$ \\
\hline
\end{tabular}

Abbreviations: $\mathrm{CI}=$ confidence interval; level 1 = clinical remission on medication; level 2 = clinical remission off oral corticosteroid medication (still on TCZ); level 3 = clinical remission off both oral corticosteroid and MTX medication (still on TCZ); level 4 = clinical remission off all anti-inflammatory medications (still on TCZ); week 0 data were taken from visit 33 (week 104) of the core study

${ }^{\text {a }}$ Exact 2-sided CI (Clopper and Pearson) based upon the observed proportion of patients 
These results are in accordance with Imagawa et al. openlabeled 48-week study of TCZ treatment in 19 pJIA patients [6]. The most common AEs were nasopharyngitis (nine events, $47.4 \%$ of patients) and upper respiratory tract infection (nine events, $47.4 \%$ ). Four patients required hospitalization due to SAEs: gastroenteritis $(n=2)$, pneumonia $(n=1)$, and sensory disturbance $(n=1)$. All laboratory abnormalities were mild in severity [6].

Horneff et al. [7] observed during 72.4 PY rare serious or medically important infections in the TCZ cohort included one case each of pneumonia, appendicitis, and influenza. No cases of TB occurred, and apart from herpes zoster, no opportunistic infections were observed. Only two patients discontinued TCZ due to intolerance. These results indicate a good tolerance of treatment that does not change during long-term administration. The awareness of AEs and treatment experience results in increased attention in observation of the patient.

As was already mentioned, the secondary objective of the study was to investigate the long-term efficacy of treatment with TCZ in patients with pJIA. All patients in this study achieved ACR70 at baseline (week 104 of the core study) and continued to achieve at least ACR50 (one patient) or ACR70 (40 patients) response up to week 24 of this longterm extension study using baseline visit of the core study. Other secondary efficacy parameters showed similar patterns of the continued response across the study. During the CHERISH core study, results were clinically meaningful because a high proportion $(89 \%)$ of patients achieved JIAACR30 response by week $16 ; 62 \%$ of patients achieved JIAACR70 response, and 26\% even achieved JIA-ACR90 response. In the double-blind, withdrawal phase, JIA flares occurred in $48.1 \%$ of patients on placebo versus in $25.6 \%$ receiving TCZ. At the end of part 2 of the study, 64.6 and $45.1 \%$ of patients receiving TCZ obtained JIA ACR 70 and JIA ACR 90 responses, respectively, which confirmed the high efficacy of the treatment. In Imagawa et al. report, the ACR Pedi 70 response rate reached $94.1 \%(16 / 17)$ at week 24 and remained high through week 48 [6].

In Horneff et al. observation [7] in 74 patients with mean treatment duration $0.98 \pm 0.59$ year in $\mathrm{TCZ}$ cohort, the improvement according to Ped ACR30/50/70/90 criteria was reached after 3 months by $61 \% / 52 \% / 35 \% / 26 \%$ patients. At 24 months, JADAS minimal disease activity was achieved in $52.4 \%$ and JADAS remission in $27.9 \%$ patients [7]. These reports also indicate the effectiveness of the therapy, but the comparison is limited due to the different duration of treatment and the method of assessment.

Presented study allowed for the acquisition of efficacy and safety data during treatment with TCZ for 131 or more weeks in children with pJIA. The potential limitation of the study is the different duration of TCZ therapy, although efficacy was assessed after the same treatment period.
The question is how long patients should receive biological agent. It is unclear whether patients with JIA who achieved remission should to continue biological treatment. Our study evaluated the efficacy of TCZ in children who did not respond to monotherapy with MTX. Guzman et al. [12] reported that the probability of attaining remission off medication in JIA within 5 years during the first 5 years of the disease was 46 57\% across JIA categories except for polyarthritis: for RFpositive $-0 \%$ and for RF-negative-14\% [12]. Moreover, based on the study by Ghiti et al., performed on adult rheumatoid arthritis (RA) patients, stopping an anti-TNF treatment was associated with substantially more flares of the disease than in RA patients with remission or with stable low disease activity continuing this biological therapy [13].

Our study demonstrated that longer duration of drug administration did not affect the incidence of AEs. Also, maintained response to the study drug was observed. The efficacy response and the consistent safety profile support the use of TCZ in pJIA. This is particularly important for new therapies in children.

\section{Conclusions}

This is the first, observational, long-term study with TCZ in pJIA in children. Our data demonstrate that continuing treatment over 104 to 131 weeks or longer with TCZ is safe and efficacious for the management of pJIA. The safety profile of TCZ in this population was consistent with that seen in the core study. The majority of AEs were unrelated to TCZ and were consistent with the pediatric and pJIA population. The presented data confirm the long-term safety of TCZ use in patients with pJIA. The efficacy response remained stable to 131 week of treatment or longer of this long-term extension study. Taken together, the continued efficacy response and the consistent safety profile support the use of TCZ in pJIA.

Acknowledgments We thank Julia Statham, MSc. ICON Clinical Research United Kingdom (UK), who worked on preparation the report from this study and David Quinlan, ICON Clinical Research (UK), who worked on clinical and statistical analyses.

We also thank all investigators (physicians, nurses, and other health professionals), who participated in the study: Poland: Anna Gruenpeter, MD, Katarzyna Kobusinska; MD; Russia: Vladimir Kelstev, MD, Alexey Sarychev, MD, and Elena Zholobova, MD.

We also thank Jacek Bil, $\mathrm{PhD}$, who provided writing and editorial services on behalf of Roche Polska Sp. z o.o.

Author contribution All authors edited and critically reviewed the manuscript drafts and approved of the final version submitted for publication. VO-W was the principal investigator, performed a literature search, and was involved in study design, collection, analysis and interpretation of data, and writing the manuscript. ZZ, EA, VC, and IN were involved in collection and interpretation of data and writing the manuscript. GD contributed to the conception and design of the study, the data interpretation, and critical revision of the manuscript. ES was involved in collection and interpretation of data, performed a literature search, and writing the manuscript. 


\section{Compliance with ethical standards}

Competing interests VO-W declares speaker's fees from Roche, Abbvie, Pfizer, outside the submitted work. Z.Ż declares no conflicts of interest. EA has received research grants from Roche, Abbott, Pfizer, Bristol-Myers Squibb, Centocor, and Novartis and received honoraria as a speaker for Roche, Novartis, and Pfizer. VCh declares no conflicts of interest. IN has received speaker's fees from Roche, AbbVie, Pfizer, Bristol-Myers Squibb, and Novartis. GD was an employee of Roche Polska Sp. z o.o. till 30.04.2015 (during the research concept and protocol development, in the time of study conduct, data analysis, and result interpretation). ES declares no conflicts of interest.

\section{Patient consent Obtained}

Ethics approval The study was conducted in accordance with the Declaration of Helsinki and good clinical practice guidelines and with local requirements.

Open Access This article is distributed under the terms of the Creative Commons Attribution 4.0 International License (http:// creativecommons.org/licenses/by/4.0/), which permits unrestricted use, distribution, and reproduction in any medium, provided you give appropriate credit to the original author(s) and the source, provide a link to the Creative Commons license, and indicate if changes were made.

\section{References}

1. Petty RE, Southwood TR, Manners P, Baum J, Glass DN, Goldenberg J, He X et al (2004) International League of Associations for Rheumatology classification of juvenile idiopathic arthritis: second revision, Edmonton, 2001. J Rheumatol 31:390-392

2. Ringold S, Seidel KD, Koepsell TD, Wallace CA (2009) Inactive disease in polyarticular juvenile idiopathic arthritis: current patterns and associations. Rheumatology (Oxford) 48: 972-977
3. Wallace CA, Huang B, Bandeira M, Ravelli A, Giannini EH (2005) Patterns of clinical remission in select categories of juvenile idiopathic arthritis. Arthritis Rheum 52:3554-3562

4. Webb K, Wedderburn LR (2015) Advances in the treatment of polyarticular juvenile idiopathic arthritis. Curr Opin Rheumatol 27:505-510

5. Brunner HI, Ruperto N, Zuber Z, Keane C, Harari O, Kenwright A et al (2015) Efficacy and safety of tocilizumab in patients with polyarticular-course juvenile idiopathic arthritis: results from a phase 3, randomised, double-blind withdrawal trial. Ann Rheum Dis 74:1110-1117

6. Imagawa $\mathrm{T}$, Yokota $\mathrm{S}$, Mori M, Miyamae T, Takei S, Imanaka H et al (2012) Safety and efficacy of tocilizumab, an anti-IL-6receptor monoclonal antibody, in patients with polyarticularcourse juvenile idiopathic arthritis. Mod Rheumatol 22:109-115

7. Horneff G, Klein A, Klotsche J et al (2016) Comparison of treatment response, remission rate and drug adherence in polyarticular juvenile idiopathic arthritis patients treated with etanercept, adalimumab or tocilizumab. Arthritis Res Ther 18:272-284

8. De Benedetti F, Brunner HI, Ruperto N, Kenwright A, Wright S, Calvo I et al (2012) Randomized trial of tocilizumab in systemic juvenile idiopathic arthritis. N Engl J Med 367:2385-2395

9. Turnier JL, Brunner HI (2016) Tocilizumab for treating juvenile idiopathic arthritis. Expert Opin Biol Ther 16:559-566

10. Giannini EH, Ruperto N, Ravelli A, Lovell DJ, Felson DT, Martini A (1997) Preliminary definition of improvement in juvenile arthritis. Arthritis Rheum 40:1202-1209

11. Wallace CA, Ruperto N, Giannini E (2004) Preliminary criteria for clinical remission for select categories of juvenile idiopathic arthritis. J Rheumatol 31:2290-2294

12. Guzman J, Oen K, Tucker LB et al (2015) The outcomes of juvenile idiopathic arthritis in children managed with contemporary treatments: results from the ReACCh-Out cohort. Ann Rheum Dis 74: $1854-1860$

13. Ghiti Moghadam M, Vonkeman HE, Ten Klooster PM, Dutch National POET Collaboration et al (2016) Stopping tumor necrosis factor inhibitor treatment in patients with established rheumatoid arthritis in remission or with stable low disease activity: a pragmatic multicenter, open-label randomized controlled trial. Arthritis Rheumatol 68:1810-1817 